\title{
Decision Support System for Quantitative Calculation of Crop Climatic Suitability in Hebei Province
}

\author{
Jing Zhang ${ }^{1,3, *}$,Youfei Zheng ${ }^{1}$, and Xin Wang ${ }^{2,3}$ \\ ${ }^{1}$ Nanjing University of Information Technology, Nanjing, Jiangsu Province, \\ P.R. China 210044 \\ ${ }^{2}$ Hebei Provincial Institute of Meteorology, Shijiazhuang, Hebei Province, P.R. China 050021 \\ ${ }^{3}$ Hebei Provincial Meteorological and Eco-environmental Laboratory, Shijiazhuang, \\ Hebei Province, P.R. China 050021 \\ Tel.: +86-311-85218904; Fax: +86-311-85218901 \\ hboxjzj@126.com
}

\begin{abstract}
The growth and development of crops would not be separated by comprehensive climatic factors, such as temperature, precipitation, sunshine and others. To some extent, the behaviors of the climate factors have great affection on the climatic suitability of crops. In order to achieve the quantitative assessment of climate factors, the decision support system for quantitative calculation on climate suitability of major crops in Hebei province such as winter wheat, corn and cotton, has been established. Developed by Microsoft Visual Basic 6.0 language, and program design built in modular structure, the system was included by three modules following as the database of climatic suitability over the stages of crops growth and development, calculation of climate suitability degerees and decision-making services. Using the differene periods such as ten days, month, season, crop growth period as the unit, the quantity changes of temperature, precipitation and sunshine would be translated into cropclimatic suitability degrees on different growth perod by membership function of fuzzy mathematics so as to achieve a quantitative assessment of climatic fators. The calculated results output adopts grid and graphic formats, and according to the results, different management decision-making information.would be chosen then .It's shown that the analysis results of crop-climatic suitability by the system for different crops and time perods are consistent with the actual situation.
\end{abstract}

Keywords: Fuzzy mathematics, Climatic Suitability, quantitative calculation, decision support systems.

Hebei Province is a major producer of winter wheat, corn and cotton; it leads the nation in terms of both land acreage and output. Growth of plant is a complex process; meteorological variables, such as temperature, precipitation, sunshine, play a vital role in the process. Research of different scales, different crop growth climate resource and ecology climate adaptability evaluation have had been done by some

* Corresponding author.

D. Li, Y. Liu, and Y. Chen (Eds.): CCTA 2010, Part I, IFIP AICT 344, pp. 381 389, 2011.

(C) IFIP International Federation for Information Processing 2011 
Chinese scholars(Lu Yu-hua et al., 2003; Bai Yongping,2000; Luo Huailiang et al., 2004; Huang Huang, 1996; Yin Dong, 2002), the suitability of only one meteorological elements for the growth of crops was also studied(Xu Xuexuan et al., 2000). In practice, when evaluating whether meteorological conditions are appropriate for crop growth and development process or not, such terms "favorable" and "unfavorable" were tended to assigh, rather vague notions. There is no clear distinction between the two conditions; thus, convey limited amount of information. In this paper, this problem would be intended to solve. The objective is to introduce a rigorous mathematical model to quantify the degree of meteorological favorability for crop growth. In addition, the exact impact of changing metrological condition on plant growth would also be revealed. All above these would provide basic data for the modernization of agricultural resources, and lay a foundation.

\section{Underlying Principles of the System}

\subsection{The Interpretation of Climate Suitability for Crops}

Crop Climate Suitability is generated by a membership function of fuzzy mathematics: numerical change in meteorological factors is the input, and the output is level of suitability for crop growth, yield and quality.

Temperature, precipitation, sunshine during crop growth period can be each treated as a separate fuzzy set $(\widetilde{T}, \widetilde{R}, \widetilde{S})$. By building membership function of fuzzy sets, i.e. suitability model, the degree of match between the variables $(t, r, s)$ and the respective fuzzy sets $\widetilde{T}(t), \widetilde{R}(r), \widetilde{S}(s)$ can be calculated, in other words, the suitability of temperature, precipitation and sunshine $(t, r, s)$ for crop growth. In this way, the climate suitability for crops can quantitatively assessed. The scale of output is from 0 to 1 : the bigger, the more desirable.

\subsection{Model of Climate Suitability for Crops}

\subsubsection{Model of Climate Suitability for Winter Wheat}

The Model of Climate Suitability for winter wheat in terms of temperature, precipitation, sunshine, as defined as follows(Ma Shuqing et al.,1994):

$$
\begin{gathered}
\tilde{T}\left(t_{i j}\right)=\frac{\left(t_{i j}-t_{l i}\right) *\left(t_{h i}-t_{i j}\right)^{B}}{\left(t_{0 i}-t_{l i}\right) *\left(t_{h i}-t_{0 i}\right)^{B}} \quad, \mathrm{~B}=\frac{t_{h i}-t_{0 i}}{t_{0 i}-t_{l i}} \\
\tilde{R}\left(r_{i j}\right)=\left\{\begin{array}{lc}
r_{i j} / r_{l i} & r_{i j}<r_{l i} \\
1 & r_{l i} \leq r_{i j} \leq r_{h i} \\
r_{h i} / r_{i j} & r_{i j}>r_{h i}
\end{array}\right.
\end{gathered}
$$




$$
\begin{gathered}
\tilde{S}\left(s_{i j}\right)= \begin{cases}e^{-\left[\left(s_{i j}-s_{0 i}\right) / b_{i}\right]^{2}} & \mathrm{~s}_{\mathrm{ij}}<s_{0 i} \\
1 & s_{i j} \geq s_{0 i}\end{cases} \\
S_{i j}=\sqrt[3]{\tilde{T}\left(t_{i j}\right) \times \tilde{R}\left(r_{i j}\right) \times \tilde{S}\left(s_{i j}\right)}
\end{gathered}
$$

Where:

$\tilde{T}\left(t_{i j}\right)$ represents climate suitability for winter wheat in terms of temperature during the ith interval of 10 days within jth month; $t_{i j}$ denotes the average temperature during the period while $t_{l i}, t_{h i}, t_{0 i}$ each indicates the lowest, highest and appropriate average temperature the winter wheat can tolerate. $\widetilde{R}\left(r_{i j}\right)$ represents climate suitability for winter wheat in terms of precipitation during the ith interval of 10 days within jth month; $r_{i j}$ denotes the amount of precipitation $(\mathrm{mm})$ during the period; we define $r_{l i}=0.6 r_{0 i}, r_{h i}=1.5 r_{0 i}$, where $r_{0 i}$ indicates the amount of water the winter wheat demands. $\tilde{S}\left(s_{i j}\right)$ represents climate suitability for winter wheat in terms of sunshine duration during the ith interval of 10 days within jth month; $s_{i j}$ denotes the total sunshine duration during the period (h); $s_{0 i}$ expresses the critical point that reach $70 \%$ of total sunshine duration for the period $(\mathrm{h}) ; b_{i}$ is a constant. $S_{i j}$ represents the comprehensive climate suitability for winter wheat.

\subsubsection{Model of Climate Suitability for Corns}

The Model of Climate Suitability for corns in terms of temperature, precipitation, sunshine, as defined as follows:

$$
\begin{gathered}
\tilde{T}\left(t_{i j}\right)=\frac{\left(t_{i j}-t_{l i}\right) *\left(t_{h i}-t_{i j}\right)^{B}}{\left(t_{0 i}-t_{l i}\right) *\left(t_{h i}-t_{0 i}\right)^{B}}, \mathrm{~B}=\frac{t_{h i}-t_{0 i}}{t_{0 i}-t_{l i}} \\
\tilde{R}\left(r_{i j}\right)= \begin{cases}r_{i j} / r_{0 i} & r_{i j}<0.7 r_{0 i} \\
1 & r_{i j} \geq 0.7 r_{0 i}\end{cases} \\
\tilde{S}\left(s_{i j}\right)= \begin{cases}s_{i j} / s_{0 i} & s_{i j}<s_{0 i} \\
1 & s_{i j} \geq s_{0 i}\end{cases} \\
S_{i j}=\sqrt[3]{\tilde{T}\left(t_{i j}\right) \times \tilde{R}\left(r_{i j}\right) \times \tilde{S}\left(s_{i j}\right)}
\end{gathered}
$$


Where:

$\tilde{T}\left(t_{i j}\right)$ represents climate suitability for corns in terms of temperature during the ith interval of 10 days within jth month; $t_{i j}$ denotes the average temperature during the period while $t_{l i}, t_{h i}, t_{0 i}$ each indicates the lowest, highest and appropriate average temperature the corns can tolerate. $\tilde{R}\left(r_{i j}\right)$ represents climate suitability for winter wheat in terms of precipitation during the ith interval of 10 days within jth month; $r_{i j}$ denotes the amount of precipitation $(\mathrm{mm})$ during the period; $r_{0 i}$ is defined as the amount of water the winter wheat demands. $\tilde{S}\left(s_{i j}\right)$ represents climate suitability for winter wheat in terms of sunshine duration during the ith interval of 10 days within $\mathrm{jth}$ month; $s_{i j}$ denotes the total sunshine duration during the period (h); $s_{0 i}$ expresses the critical point that reach $70 \%$ of total sunshine duration for the period $(\mathrm{h}) ; b_{i}$ is a constant. $S_{i j}$ represents the comprehensive climate suitability for winter wheat.

\subsubsection{Model of Climate Suitability for Cottons}

The Model of Climate Suitability for cottons in terms of temperature, precipitation, sunshine, as defined as follows:

$$
\begin{gathered}
\tilde{T}\left(t_{i j}\right)=\frac{\left(t_{i j}-t_{l i}\right) *\left(t_{h i}-t_{i j}\right)^{B}}{\left(t_{0 i}-t_{l i}\right) *\left(t_{h i}-t_{0 i}\right)^{B}} \quad, \mathrm{~B}=\frac{t_{h i}-t_{0 i}}{t_{0 i}-t_{l i}} \\
\tilde{R}\left(r_{i j}\right)=\left\{\begin{array}{lc}
r_{i j} / r_{l i} & r_{i j}<r_{l i} \\
1 & r_{l i} \leq r_{i j} \leq r_{h i} \\
r_{h i} / r_{i j} & r_{i j}>r_{h i}
\end{array}\right. \\
\tilde{S})=\left\{\begin{array}{lc}
e^{\left[-\left(s_{i j}-s_{0 i}\right) / b_{i}\right]^{2}} & (\text { sowing and } \\
e^{-\left[\left(s_{i j}-s_{0 i}\right) / b_{i}\right]^{2}} & (o \text { therwise })
\end{array}\right. \\
S_{i j}=\sqrt[3]{\widetilde{T}\left(t_{i j}\right) \times \widetilde{R}\left(r_{i j}\right) \times \tilde{S}\left(s_{i j}\right)}
\end{gathered}
$$

Where:

$\tilde{T}\left(t_{i j}\right)$ represents climate suitability for cottons in terms of temperature during the ith interval of 10 days within jth month; $t_{i j}$ denotes the average temperature during the period while $t_{l i}, t_{h i}, t_{0 i}$ each indicates the lowest, highest and appropriate 
average temperature the corns can tolerate. $\widetilde{R}\left(r_{i j}\right)$ represents climate suitability for winter wheat in terms of precipitation during the ith interval of 10 days within jth month; $r_{i j}$ denotes the amount of precipitation $(\mathrm{mm})$ during the period; $r_{l i}, r_{h i}$ are defined as the lower and upper limit, respectively, of amount of water cotton demands. $\tilde{S}\left(s_{i j}\right)$ represents climate suitability for winter wheat in terms of sunshine duration during the ith interval of 10 days within jth month; $s_{i j}$ denotes the total sunshine duration during the period (h); $s_{0 i}$ expresses the critical point that reach $70 \%$ of total sunshine duration for the period (h); $b_{i}$ is a constant. $S_{i j}$ represents the comprehensive climate suitability for winter wheat.

\subsubsection{Calculating Climate Suitability for Different Time Intervals}

From sowing to harvesting, the growth of crops spans different months, quarters or even growth stages. Climate suitability of months, quarters or growth stages corresponds to individual 10-day climate suitability. The subject of system is crops; based on 10-day climate suitability collected from individual weather stations, the climate suitability for different time intervals would be derived by taking the weighted average of 10-day climate suitability(Huang Huang, 1996; Zhao Feng et al.,2003).

$$
\begin{gathered}
\tilde{T}\left(t_{m j}\right)=\sum_{j=m_{1}}^{m_{2}} b_{t i} \tilde{T}\left(t_{i j}\right) \\
\tilde{R}\left(r_{m j}\right)=\sum_{j=m_{1}}^{m_{2}} b_{r i} \tilde{R}\left(r_{i j}\right) \\
\tilde{S}\left(s_{m j}\right)=\sum_{j=m_{1}}^{m_{2}} b_{s i} \tilde{S}\left(s_{i j}\right) \\
S_{m j}=\sqrt[3]{\widetilde{T}\left(t_{m j}\right) \times \tilde{R}\left(r_{m j}\right) \times \tilde{S}\left(s_{m j}\right)}
\end{gathered}
$$

Where:

$\tilde{T}\left(t_{\mathrm{m} j}\right), \tilde{R}\left(r_{\mathrm{m} j}\right), \tilde{S}\left(s_{m j}\right)$ are the climate suitability in terms of temperature, precipitation, sunshine duration, respectively, during mth month of crop growth season in jth year; $m_{1}, m_{2}$ indicates the beginning and ending 10-day interval of mth month (within a quarter or a growth season. In the case that $m_{1}=1$ and $m_{2}=3, \widetilde{T}\left(t_{\mathrm{m} j}\right)$, $\tilde{R}\left(r_{\mathrm{m} j}\right), \tilde{S}\left(s_{m j}\right)$ each corresponds to the monthly climate suitability in terms of temperature, precipitation, sunshine duration, respectively. $b_{t j}, b_{r j}, b_{s j}$ denote the weight assigned to climate suitability in terms of temperature, precipitation, sunshine 
duration, respectively, of the $i$ th 10-day interval. $\tilde{T}\left(t_{t j}\right), \tilde{R}\left(r_{t j}\right), \tilde{S}\left(s_{t j}\right)$ represent the 10-day climate suitability in terms of temperature, precipitation, sunshine duration, respectively. Finally, $s_{m j}$ is the comprehensive climate suitability for mth month within jth year.

\section{Introduction of System}

\subsection{System Operating Environment}

The system is developd by Microsoft Visual Basic 6.0, the development platform is Chinese version of Windows XP/2003 Server, the operating system applied is Windows 32-bit desktop operation system. The hardware environment: desktop PC based on Intel's 808x instruction system. Software development environment: Windows XP/2003 Server based operating system.

\subsection{System Structure}

The program employs modular structure and drop-down menu. The program consists of three components(Fig.1): a database that records the weather parameters that determine climate suitability for crops, a calculation module and a decision making module. The calculation module also includes two sub-components: one accommodates real-time climate suitability while the other provides the historical information.

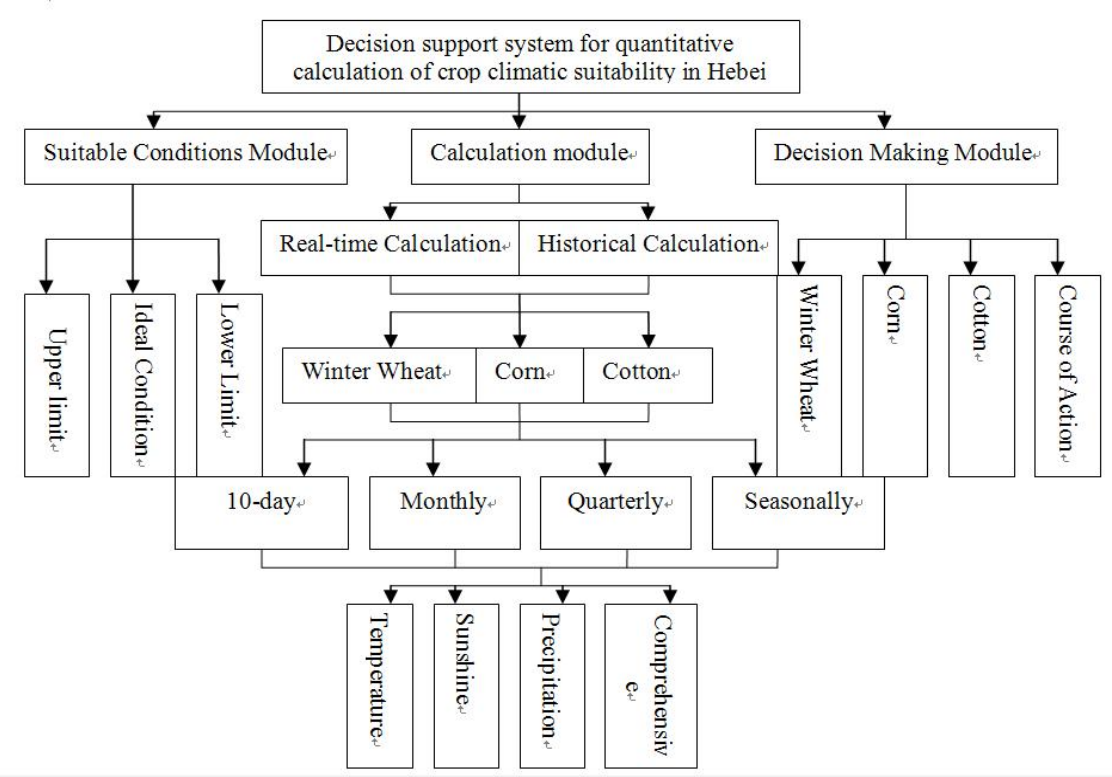

Fig. 1. Structure of Decision support system for quantitative calculation of crop climatic suitability in Hebei 
The real-time calculation module is capable of produce climate suitability based on different time horizons, i.e., every 10 days, monthly, quarterly or for the entire growth period. The decision making module provides background information for crop growth and make suggestions on appropriate management measures.

\subsection{System Function}

\subsubsection{Climate Suitability Condition Management}

The database contains all kinds of agro-meteorological indicators of winter wheat, corn, cotton that spans various growth stages from planting to harvest: including target climate suitability, upper and lower bound of a range of acceptable climate suitability level. Researchers may choose to consult, modify, expand or streamline the database.

\subsubsection{Calculation of Real-Time Climate Suitability}

The system receives and processes real-time meteorological information on a 10-day basis. Based on the comprehensive meteorological data, 10-day climate suitability in terms of temperature, precipitation, sunshine or combined could be calculated, pertaining to winter wheat, corn or cotton. From that, the same indicators on monthly or quarterly basis could be further obtained, or even for the entire growth season. The output is presented in graph and spreadsheet.

\subsubsection{Calculation of Historical Climate Suitability}

The system can read the historical metrological information including temperature, precipitation, sunshine from the database to calculate the climate suitability in terms of temperature, precipitation, sunshine or combined at different point in time. The result can form the foundation for the analyzing the impact of climate change on agriculture.

\subsubsection{Decision Making Module}

According to the calculation, the system can make recommendations on course of actions in response to varying weather conditions. Researchers can consult, modify, add or delete the recommendations; researches can also search information related to the plant growth and development.

\subsection{System Operating Procedures}

The system can automatically collect real-time 10-day weather data from weather stations across the province. The system can process the data and extract the relevant information, including temperature, precipitation and sunshine. The selected data is then incorporated into the database and become the input of function (1) (12) to derive climate suitability indicators. The procedure is illustrated in Fig.2.

The output is presented in graphs or tables.

Table output: By applying MSHFlexGrid Control. the format would be set up, so that the system can export the climate suitability in terms of temperature, precipitation, sunshine or combined for different areas during different time intervals; 


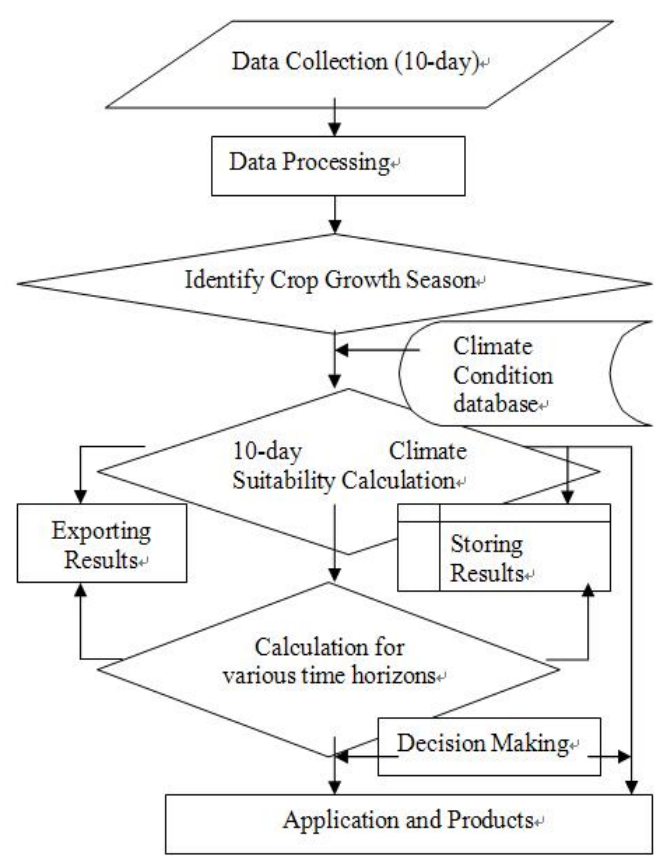

Fig. 2. System operating procedures

\subsection{Characteristics of the System}

The system allows real-time calculation of climate suitability for crops. Individual modules of the system run relatively independently from each other, making way for future modification or maintenance. The system can be easily expanded; the interface is user-friendly and is very easy to use. In addition, in consideration of possible misuse of the system, a dialog box is developed to help researchers tackle errors or mistakes they made. In conclusion, the system could be believed widely adopted.

\section{Conclusion}

The system is developed by VB6.0 language; it employed a modular structure which is easy to maintain and expand. The system works in a time frame of 10day, month, quarter or growth season; it uses the membership function of fuzzy math to convert meteorological data, such as the numerical change in temperature, precipitation or sunshine into climate suitability for crops. The result, which is exported in various formats, can provide quantitative basis for agriculture decision making. The practical application showed that has very good business application effect. 


\section{References}

Lu, Y., Zheng, D.: Evaluation on eco-climate adaptability of crop and herbage in northern ecotone. Chinese Journal of Eco-Agriculture 11(4), 130-133 (2003)

Bai, Y.: The assessment and quantization of agricultural ecoclimate resources in Northwest region (Gansu, Ningxia). Journal of Natural Resources 15(3), 218-224 (2000)

Luo, H., Chen, G., et al.: Eco-climatic suitability of agricultural research. Agricultural Resources and Regional Planning 25(1), 28-32 (2004)

Huang, H.: Red and yellow earth of China's climatic and ecological adaptability of crop production research. Journal of Natural Resources 11(4), 341-345 (1996)

Yin, D., Wang, C.: The evaluation model of climatic resources of herbage in the pastoral area of northern China. Journal of Natural Resources 17(4), 494-497 (2002)

Xu Xue-xuan, X., Gao, P., et al.: Fuzzy Analysis on Rainfall Adaptability to Crop Growth in Yan' an Area. Soil and Water Conservation 7(2), 73-76 (2000)

Ma, S.: Jilin Agricultural Climate Research, p. 33. Meteorological Press, Beijing (1994)

Zhao, F., Qian, H.-s., et al.: The climatic suitability model of crop:a case study of winter wheat in henan province. Resources Science 25(6), 80-81 (2003) 